\section{Arkivoc

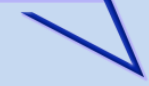

Archive for

Organic Chemistry
The Free Internet Journal

for Organic Chemistry
Paper

Arkivoc 2020, part vi, 209-219

\title{
Completely dissimilar: The reactivity of 1-unsubstituted 3-chloroquinoline-2,4- diones with ethylene diamine and ethanolamine to form new molecular rearrangements
}

\section{Antonin Klásek, ${ }^{* a}$ Antonin Lyčka, ${ }^{b}$ and Michal Rouchal ${ }^{\mathrm{a}}$}

${ }^{a}$ Department of Chemistry, Faculty of Technology, Tomas Bata University, CZ-762 72 Zlín, Czech Republic ${ }^{b}$ University of Hradec Králové, Faculty of Science, Rokitanského 62, CZ-500 03 Hradec Králové 3, Czech Republic E-mail: klasek@ft.utb.cz

\section{Abstract}

3-Chloroquinoline-2,4-diones react with ethanolamine to give 3-hydroxyethylaminoquinoline-2,4-diones. By reacting with isothiocyanic acid, these compounds cyclize to form thioxoimidazo derivatives. If a benzyl group is attached to carbon atom C-3, it is cleaved off. Simultaneously, molecular rearrangement proceeds through the formation of compounds with quinazoline skeletons. However, when using ethylene diamine, the compounds are subject to new types of molecular rearrangement leading to the formation of new quinazoline derivatives.<smiles>[R]C1(NCCO)C(=O)Nc2ccccc2C1=O</smiles>

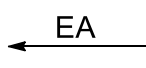<smiles>[R]C1(Cl)C(=O)Nc2ccccc2C1=O</smiles><smiles>[R]C(=O)NCCn1c(=O)[nH]c2ccc(C=[R10]3ccccc3)cc2c1=O</smiles><smiles>O=C1Nc2ccccc2C2(C(=O)c3ccccc3)NCCN12</smiles>

Keywords: $\alpha$-Aminoketones, indolones, quinazolinones, quinolinediones, rearrangement 


\section{Introduction}

Many biologically active compounds contain the amino functional groups. In the quinolinediones series, which is our particular area of interest, we managed to prepare 3-amino derivatives using 3-chloroquinolinediones and ammonium salts or primary amines. ${ }^{1}$ Prior to the publication of our results, only two 3aminoquinolinedione were previously known. The first 3 -aminoquinolinedione was prepared by acid hydrolysis of the corresponding 3-acetamido derivative, which was obtained by rearranging the 6-chloro-4hydroxy-3-imino-1-methyl-4-o-phenyl-3,4-dihydro-1H-quinolin-2-one molecule. ${ }^{2}$ The ammonolysis of 3chloroquinolinediones with aqueous ammonia led to formation of only one product bearing the 3-benzyl substituent at the $\mathrm{C}-3$ atom. ${ }^{3}$

The biological activity of 3-aminoquinoline-2,4-diones was described for 3-amino-3-(4-fluorophenyl)-1H-quinoline-2,4-dione, which is effective against oxidative stress-related diseases ${ }^{4}$ and inhibits cisplatin-induced hearing loss by the suppressing the reactive oxygen species. ${ }^{5,6} \mathrm{~A}$ similar effect was exhibited by 3-amino-6-fluoro-3-(4-fluoro-phenyl)- $1 \mathrm{H}$-quinoline-2,4-dione. ${ }^{4}$

We discovered that 3 -aminoquinolinediones are subject to molecular rearrangement through their reactions with isocyanic acid, forming from urea in boiling acetic acid. By using this synthetic method, new imidazoquinazolinediones, 3-acylureidoindolones, spiro-imidazolidine-indolediones and dihydroimidazoquinolones were prepared. ${ }^{7,8}$ We also found that the preferable sources of isocyanic and isothiocyanic acids are sodium cyanate and potassium thiocyanate, respectively, both in acetic acid. Under these conditions, more types of new heterocycles were prepared, e.g., thioureidooxindoles, spiro-oxindoles, dihydroimidazoquinolones and their sulfur analogues. ${ }^{9}$

In order to discover the influence of other substituents in the amine molecule, we chose to use ethanolamine, as it is an as easily available and inexpensive compound. In our earlier paper, ${ }^{10}$ we described its reaction with 3-chloroquinoline-2,4-diones. In line with our expectations, $\mathrm{N}$-substituted compounds $\mathbf{2}$ were obtained and their reaction with isocyanic acid afforded three new types of heterocycles.

In this paper, we describe the analogous reactions, however, beginning with 1-unsubstituted 3chloroquinoline-2,4-diones 1 and ethylene diamine (EDA) or ethanolamine (EA).

\section{Results and Discussion}

Compounds $\mathbf{2} \mathbf{b}-\mathbf{2} \mathbf{d}$ were prepared according to literature ${ }^{1}$ new compound $\mathbf{2} \mathbf{a}$ was prepared now. For NMR data of compound 2a see Table 1. The reaction of 1-substituted 3-(2-hydroxyethylamino)quinoline-2,4-diones (2a-d) with isothiocyanic acid was carried out using potassium thiocyanate in acetic acid. Four different compounds $\mathbf{3}-\mathbf{6}$ were isolated (Scheme 1, NMR Table 1). Compound $\mathbf{3 c}$ and $\mathbf{6 d}$ were created by adding HNCS to the corresponding compounds $\mathbf{2 c}$ and $\mathbf{2 d}$ without rearrangement. Compound $\mathbf{4} \mathbf{c}$ is the product of the debenzylation of $3 c$. Previous studies have shown the easy debenzylation of compounds bearing a benzyl group in position $3 .{ }^{9,11}$ Compounds $\mathbf{5 a}, \mathbf{5 b}$ and $\mathbf{5} \mathbf{d}$ are products of a molecular rearrangement of compounds $\mathbf{2}$, in which the quinolone ring opens to form an intermediate isocyanate, before subsequently closing and forming a quinazoline ring. Compounds $\mathbf{5}$ are structurally similar to compounds prepared in previous studies ${ }^{9}$ and their structure was established on the basis of 2D-NMR experiments (see Table 2). 


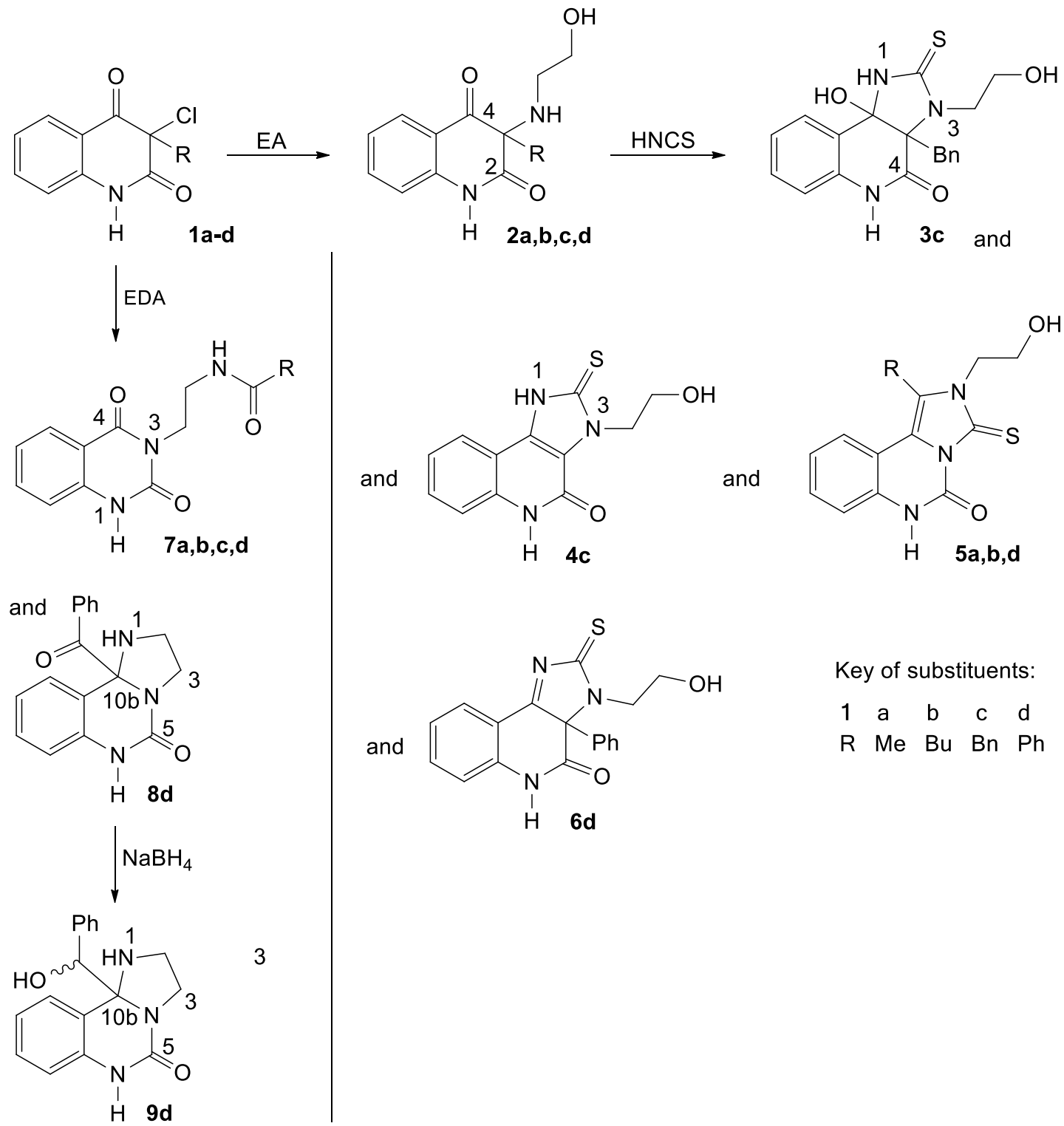

Scheme 1. Reaction of 3-chloroquinolinediones with ethanolamine and ethylene diamine.

The results of the reactions of chloro-derivatives 1a-d with ethylene diamine were surprising. We obtained two types of compounds that did not react with isocyanic and isothiocyanic acids, contrary to previous studies. From their 2D-NMR spectra (see Tables 2 and 3), the structures of $\mathbf{7}$ and $\mathbf{8}$ were established. A key piece of information concerning the constitution of compounds 7 was obtained from the ${ }^{1} \mathrm{H}-{ }^{15} \mathrm{~N} \mathrm{HMBC}$ spectra, where a correlation of the $\mathrm{CON}(1) \mathrm{H}$ proton with $=\mathrm{N}(3)$ - nitrogen typical of $\mathrm{NH}-\mathrm{X}-\mathrm{N}$ fragments was detected. ${ }^{13} \mathrm{C}$ Chemical shifts of $\mathrm{C}(\mathrm{O}) \mathrm{NH}$ in compounds 1 were shifted to ca. $150 \mathrm{ppm}$, characteristic of a urea fragment (-N$\mathrm{C}(=\mathrm{O})-\mathrm{N}-)$ in the compounds 7. Moreover, correlations of the second carbonyl group $(\mathrm{C}(4)=\mathrm{O})$ with aromatic protons from peri- position $(\mathrm{H}-5)$ were observed in gs ${ }^{1} \mathrm{H}-{ }^{13} \mathrm{C} \mathrm{HMBC}$ spectra. The last correlation from the peri position was missing in from ${ }^{1} \mathrm{H}-{ }^{13} \mathrm{C} \mathrm{HMBC}$ spectrum of compound $\mathbf{8 d}$, where a correlation of this proton with quaternary carbon $10 \mathrm{~b}$ was observed. Through-space interactions of $\mathrm{H}-10$ proton with ortho and meta protons of phenyl group were detected in the NOESY spectrum of compound $\mathbf{8 d}$. Correlations were also found in the $\mathrm{C}_{6} \mathrm{H}_{5} \mathrm{CO}$ fragment, both in the ${ }^{1} \mathrm{H}^{-13} \mathrm{C} \mathrm{HMQC}$ and ${ }^{1} \mathrm{H}_{-}{ }^{13} \mathrm{C} \mathrm{HMBC}$ spectra. 
To the best our knowledge, compound 8, as well as its analogues bearing an acyl group in position 10b, have not been described in the literature. Compounds 7 are also unknown, but many of their analogues containing modified amino groups are constituents of serotonin antagonists, e.g., ketanserin. ${ }^{12}$ They are also a part of dsDNA binding unnatural oligopeptides. ${ }^{13}$

Our proposal of the reaction mechanism is shown (Scheme 2). We assumed, that this unprecedented rearrangement of compounds 1 is caused by the presence of ethylene diamine, which is much more basic then ethanolamine. One of the reaction product (8d) contains an imidazolidine ring. The presence of both nitrogen atoms at one quaternary carbon atom is only possible when the entire of ethylene diamine molecule is added to the carbonyl group. However, formerly must be removed the halogen atom to the prevention of nucleophilic substitution at C-3 atom. The formation of 3-hydroxyquinolinedione as the first intermediate is unsurprising, as its conversion to intermediate $\mathbf{A}$. The following base catalyzed opening of the ring $B$ leads to the formation of isocyanate intermediate $\mathbf{B}$, which after the intramolecular addition of an $\mathrm{NH}$ group produces

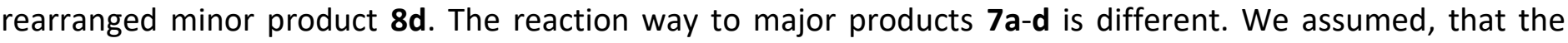
substitution of ethylene diamine for a chlorine atom in $\mathbf{1 a - d}$ following cyclization leads to intermediate $\mathbf{C}$, which is a common reaction in the chemistry of quinolinediones. By the addition of a molecule of water

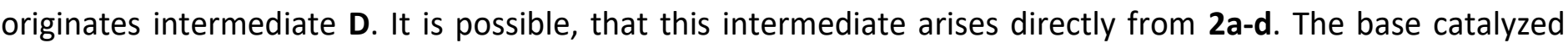
opening of the ring $B$ in intermediate $\mathbf{D}$ and, along with the addition of water, leads to intermediate $\mathbf{E}$, which cyclizes to form the major products $7 \mathbf{a}-\mathbf{d}$.

The experiments relating to the hydrolysis of compounds 7 were unsuccessful. Using aqueous sodium hydroxide, only non-separable mixtures of compounds were obtained. The same results were obtained using non-aqueous conditions, ${ }^{14}$ or using sodium peroxide as a mild hydrolysing agent. ${ }^{15}$ On the other hand, we were successful in reducing ketone $\mathbf{8 d}$. Compound $\mathbf{8 d}$ was reduced by $\mathrm{NaBH}_{4}$ to give compound $\mathbf{9 d}$. The carbonyl group from the $\mathrm{C}_{6} \mathrm{H}_{5} \mathrm{CO}$ fragment disappeared in the ${ }^{13} \mathrm{C} \mathrm{NMR}$ spectrum and new resonance signals were detected in compound $\mathbf{9 d}$ at 77.3 and $74.7 \mathrm{ppm}$, respectively. Those belonged to two $\mathrm{CHOH}$ moieties (diastereoisomers as a consequence of two stereogenic centers existing in compound 9d). Through-space interactions of $-\mathrm{CH}(-) \mathrm{O}$ - protons with $\mathrm{OH}$ and $\mathrm{NH}$ protons, as well as ortho protons of phenyl groups, were detected in the NOESY spectrum of compound 9d. However, the results of NOESY were multivalent, because the conformation is not fixed. As a consequence of the free rotation around $\mathrm{C}(\mathrm{q})-\mathrm{CHOH}$, protons of both diastereoisomers can be located side- by -side. The constitution of compound $9 \mathbf{d}$ retrospectively confirms the constitution of compound $8 \mathbf{d}$. The data for ${ }^{1} \mathrm{H},{ }^{13} \mathrm{C}$ and ${ }^{15} \mathrm{~N} N \mathrm{NM}$ are shown in Tables 2 and 3.

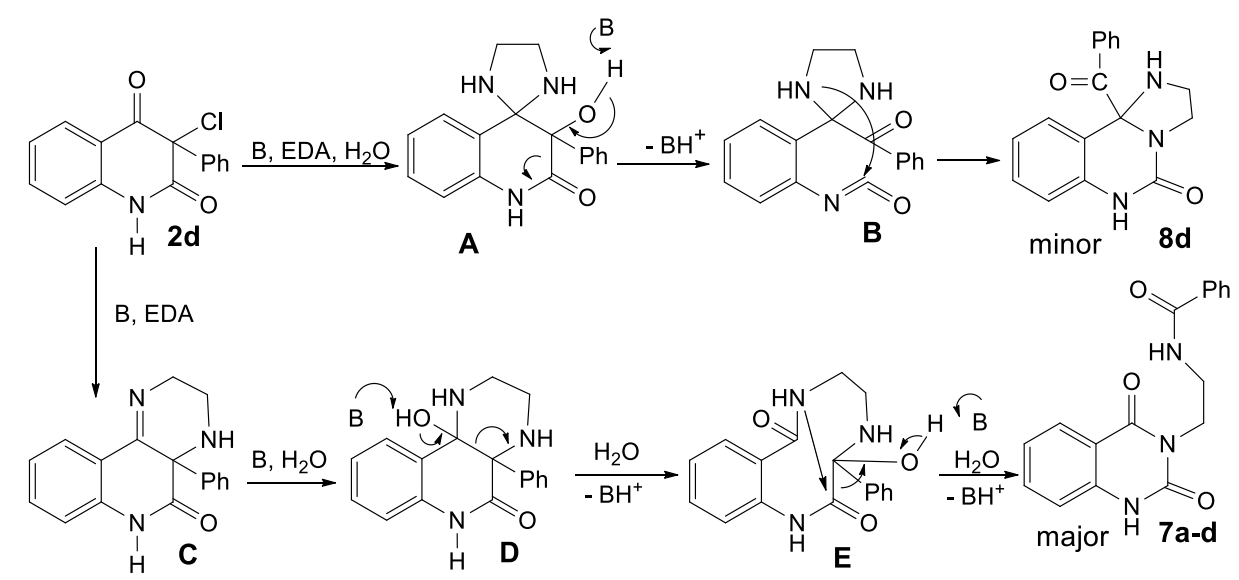

Scheme 2. Proposed mechanism for the rearrangement of compound $\mathbf{2 d}$. 
Table 1. ${ }^{1} \mathrm{H},{ }^{13} \mathrm{C}$ and ${ }^{15} \mathrm{~N}$ NMR data $(\delta, \mathrm{ppm})$ of compounds $2 \mathrm{a}, 3 \mathrm{c}, \mathbf{4 c}$ and $\mathbf{6 d}$ in DMSO- $d_{6}$

\begin{tabular}{|c|c|c|c|c|c|c|c|c|}
\hline \multirow[t]{2}{*}{ Position } & \multicolumn{2}{|c|}{$2 a$} & \multicolumn{2}{|c|}{$3 c$} & \multicolumn{2}{|c|}{$4 c$} & \multicolumn{2}{|c|}{$6 d$} \\
\hline & $\delta_{H}$ & $\delta_{c}$ & $\delta_{H}$ & $\delta_{C}$ & $\delta_{H}$ & $\delta_{c}$ & $\delta_{H}$ & $\delta_{c}$ \\
\hline 1 & 10.90 & $-248.4^{a}$ & 9.19 & $-236.9^{\mathrm{acb}}$ & 13.8 & $-222.3^{\mathrm{a}, \mathrm{e}}$ & 9.40 & - \\
\hline 2 & - & 173.1 & - & 181.4 & - & 152.9 & - & 184.2 \\
\hline 3 & - & 68.8 & - & $-256.2^{a}$ & - & $-220.5^{a}$ & - & - \\
\hline $3 a$ & - & - & - & 72.8 & - & 109.5 & - & 78.5 \\
\hline 4 & - & 196.3 & - & 168.4 & - & 166.9 & - & 168.2 \\
\hline $4 a$ & - & 118.5 & - & - & - & - & - & - \\
\hline 5 & 7.76 & 127.0 & 10.29 & $-243.9^{a, c}$ & 11.93 & $-232.4^{a, f}$ & 10.21 & - \\
\hline $5 a$ & - & - & - & 134.0 & - & 136.2 & - & 134.6 \\
\hline 6 & 7.11 & 122.6 & 6.37 & 114.3 & 7.41 & 116.0 & 6.99 & 115.3 \\
\hline 7 & 7.60 & 136.2 & 6.95 & 129.0 & - & - & 7.33 & 128.9 \\
\hline 8 & 7.09 & 116.3 & 6.90 & 122.2 & 7.26 & 122.4 & 7.01 & 122.8 \\
\hline $8 a$ & - & 141.7 & - & - & - & - & - & - \\
\hline $\mathrm{N}^{\mathrm{b}} \mathrm{HCH}_{2}$ & 2.39 & 46.7 & - & - & - & - & - & - \\
\hline $\mathrm{CH}_{2} \mathrm{O}$ & 3.41 & 61.0 & - & - & - & - & - & - \\
\hline 9 & - & - & 7.55 & 126.0 & 8.03 & 121.6 & 7.50 & 127.7 \\
\hline $9 a$ & - & - & - & 122.1 & - & 118.3 & - & 121.4 \\
\hline $9 b$ & - & - & - & 85.8 & - & 132.6 & - & 87.1 \\
\hline $\mathrm{OH}$ & - & - & 6.16 & - & - & - & 6.76 & - \\
\hline \multirow[t]{2}{*}{$\mathrm{NCH}_{2}$} & - & - & 4.03 & 46.9 & 4.55 & 46.3 & 3.99 & 48.8 \\
\hline & & & & & & & 3.67 & \\
\hline \multirow[t]{2}{*}{$\mathrm{CH}_{2}$} & - & - & 3.73 & 59.2 & 3.72 & 58.5 & 3.67 & 58.4 \\
\hline & & & 3.64 & & & & 3.41 & \\
\hline $\mathrm{OH}$ & 4.48 & - & 4.66 & - & - & - & 4.57 & - \\
\hline \multirow[t]{2}{*}{$1(R)$} & 1.37 & 25.2 & 3.37 & 36.1 & - & - & - & 131.9 \\
\hline & & & 3.18 & & & & & \\
\hline $2(R)$ & - & - & - & 132.5 & - & - & 7.33 & 128.0 \\
\hline $3(R)$ & - & - & 7.05 & 127.1 & - & - & 7.33 & 128.0 \\
\hline $4(R)$ & - & - & 6.90 & 130.5 & - & - & 7.33 & 131.5 \\
\hline $5(R)$ & - & - & 6.93 & 126.6 & - & - & - & - \\
\hline
\end{tabular}

\section{Conclusions}

In conclusion, $\mathrm{N}$-unsubstituted 3-chloroquinolinediones $\mathbf{1}$ reacts with ethanolamine to give 3hydroxyethylaminoquinolinediones $\mathbf{2}$. These compounds cyclize with isothiocyanic acid to form thioxoimidazo derivatives. If a benzyl group is attached to carbon atom C-3, it is cleaved off owing to the high acidity of isothiocyanic acid. Simultaneously, molecular rearrangement proceeds under the formation of a quinazoline skeleton. However, when using the more basic ethylene diamine, compounds 1 are subject to new types of molecular rearrangement leading to the new quinazoline derivatives $\mathbf{7}$ and $\mathbf{8}$. 
This paper's significant contribution has been the discovery of these two new compounds, which can be used in synthetic organic chemistry. Compounds $\mathbf{4}$ and $\mathbf{5}$ can also be used as a precursor in the preparation of hard blocks in order to modify the polymeric properties, e.g., enhanced thermostability or reduced combustibility. ${ }^{16}$

Table 2. ${ }^{1} \mathrm{H},{ }^{13} \mathrm{C}$ and ${ }^{15} \mathrm{~N} N M R$ data $(\delta, \mathrm{ppm})$ of compounds $\mathbf{5 a}, \mathbf{5 b}, \mathbf{5 d}, \mathbf{8 d}$ and $\mathbf{9 d}$ in DMSO- $d_{6}$

\begin{tabular}{|c|c|c|c|c|c|c|c|c|c|c|c|c|}
\hline \multirow{2}{*}{$\begin{array}{l}\text { Posi } \\
\text { tion }\end{array}$} & \multicolumn{2}{|c|}{$5 a$} & \multicolumn{2}{|c|}{$5 b$} & \multicolumn{2}{|c|}{$5 d$} & \multicolumn{2}{|r|}{$8 d$} & \multicolumn{2}{|c|}{ 9d major } & \multicolumn{2}{|c|}{ 9d minor } \\
\hline & $\delta_{H}$ & $\delta_{c}$ & $\delta_{H}$ & $\delta_{c}$ & $\delta_{H}$ & $\delta_{c}$ & $\delta_{H}$ & $\delta_{c}$ & $\delta_{H}$ & $\delta_{c}$ & $\delta_{H}$ & $\delta_{c}$ \\
\hline \multirow[t]{2}{*}{1} & - & 122.0 & - & 126.1 & - & 127.9 & 4.40 & $-318.7^{a}$ & 3.20 & $-322.8^{a}$ & n.o. & - \\
\hline & & & & & & & & & & & & $323.2^{\mathrm{a}}$ \\
\hline \multirow[t]{2}{*}{2} & - & - & - & $-203.6^{a}$ & - & $-204.4^{\mathrm{a}}$ & 3.34 & 43.3 & 3.61 & 45.5 & 3.56 & 45.4 \\
\hline & & & & & & & 2.86 & & 3.22 & & 3.44 & \\
\hline \multirow[t]{2}{*}{3} & - & 160.0 & - & 160.1 & - & 160.2 & 3.51 & 43.7 & 3.18 & 43.2 & 3.13 & 42.4 \\
\hline & & & & & & & 3.30 & & 2.96 & & 3.01 & \\
\hline \multirow[t]{2}{*}{4} & - & - & - & $-203.6^{a}$ & - & $-203.0^{a}$ & - & $-275.7^{a}$ & - & $-277.4^{a}$ & - & - \\
\hline & & & & & & & & & & & & $276.9^{\mathrm{a}}$ \\
\hline 5 & - & 144.8 & - & 144.7 & - & 144.8 & - & 150.8 & - & 151.3 & - & 151.0 \\
\hline \multirow[t]{3}{*}{6} & 10.96 & - & 11.01 & $-262.1^{a . b}$ & 11.15 & $-261.7^{a, c}$ & 9.48 & $-270.4^{\mathrm{a}, \mathrm{d}}$ & 8.96 & $-271.2^{\mathrm{a}, \mathrm{g}}$ & 8.75 & - \\
\hline & & & & & & & & & & & & $271.7^{\mathrm{a}}$ \\
\hline & & & & & & & & & & & & ,h \\
\hline $6 a$ & - & 134.0 & - & 134.0 & - & 134.3 & - & 137.9 & - & 137.7 & - & 137.5 \\
\hline 7 & 7.03 & 114.9 & 7.08 & 115.0 & 7.05 & 115.2 & 6.84 & 114.1 & 6.64 & 112.8 & 6.56 & 112.5 \\
\hline 8 & 7.24 & 128.1 & 7.29 & 128.2 & 7.22 & 127.9 & 7.16 & 129.6 & 7.06 & 128.3 & 7.08 & 128.4 \\
\hline 9 & 7.09 & 123.0 & 7.15 & 123.2 & 6.79 & 122.6 & 6.82 & 121.2 & 6.72 & 119.8 & 6.82 & 120.0 \\
\hline 10 & 7.71 & 122.2 & 7.63 & 121.8 & 6.56 & 121.2 & 7.33 & 126.4 & 6.68 & 126.6 & 7.10 & 126.9 \\
\hline 10a & - & 112.9 & - & 112.6 & - & 112.2 & - & 117.5 & - & 120.9 & - & 119.9 \\
\hline $10 b$ & - & 118.8 & - & 118.8 & - & 120.2 & - & 86.4 & - & 83.0 & - & 82.9 \\
\hline $1^{\prime}(R$ & 2.59 & 11.2 & 3.04 & 23.6 & - & 124.5 & - & $195.5^{\mathrm{e}}$ & $4.57^{\mathrm{j}}$ & $77.3^{\mathrm{j}}$ & $4.53^{\mathrm{j}}$ & $74.7^{\mathrm{j}}$ \\
\hline \multicolumn{13}{|l|}{$\left.{ }^{1}\right)$} \\
\hline $2^{\prime}(\mathrm{R}$ & - & - & 1.57 & 29.6 & 7.65 & 128.8 & - & $134.3^{f}$ & - & $140.9^{f}$ & - & $140.3^{f}$ \\
\hline \multicolumn{13}{|l|}{$\left.{ }^{1}\right)$} \\
\hline $3^{\prime}(R$ & - & - & 1.46 & 21.9 & 7.59 & 131.5 & 8.24 & $129.8^{f}$ & 6.98 & $127.8^{f}$ & 6.83 & $127.6^{f}$ \\
\hline \multicolumn{13}{|l|}{$\left.{ }^{1}\right)$} \\
\hline $4^{\prime}(R$ & - & - & 0.97 & 13.7 & 7.65 & 129.6 & 7.42 & $128.3^{f}$ & 7.12 & $126.6^{f}$ & 7.06 & $126.9^{f}$ \\
\hline \multicolumn{13}{|l|}{$\left.{ }^{1}\right)$} \\
\hline $\mathrm{N}(2$ & 4.23 & 46.2 & 4.21 & 46.1 & 3.91 & 46.2 & 7.54 & $132.8^{f}$ & 7.18 & $126.7^{f}$ & 7.14 & $127.0^{f}$ \\
\hline \multicolumn{13}{|l|}{) $\mathrm{CH}$} \\
\hline \multicolumn{13}{|l|}{2} \\
\hline OC & 3.70 & 57.6 & 3.75 & 57.4 & 3.58 & 56.6 & - & - & - & - & - & - \\
\hline \multicolumn{13}{|l|}{$\mathrm{H}_{2}$} \\
\hline $\mathrm{OH}$ & 4.98 & - & 4.97 & - & 4.83 & - & - & - & 5.60 & - & 5.33 & - \\
\hline
\end{tabular}

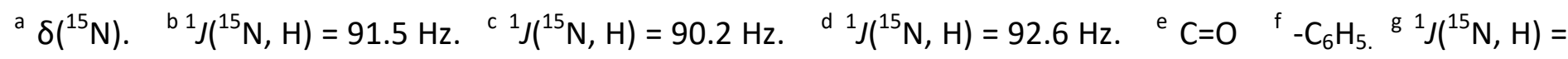
$92.5 \mathrm{~Hz} . \quad{ }^{\mathrm{h}}{ }^{1} \mathrm{~J}\left({ }^{15} \mathrm{~N}, \mathrm{H}\right)=92.2 \mathrm{~Hz}$. ${ }^{\text {i }}$ diastereoisomeric ratio $1: 0.34 .{ }^{\mathrm{j}} \mathrm{CHOH}$ 


\section{Experimental Section}

General. Melting points were determined on a Kofler block. IR $(\mathrm{KBr})$ spectra were recorded on a Smart OMNITransmission Nicolet iS10 spectrophotometer. The ${ }^{1} \mathrm{H},{ }^{13} \mathrm{C}$, and ${ }^{15} \mathrm{~N}$ NMR spectra were recorded on a Bruker Avance III HD 500 spectrometer $\left(500.13 \mathrm{MHz}\right.$ for ${ }^{1} \mathrm{H}, 125.76 \mathrm{MHz}$ for ${ }^{13} \mathrm{C}$, and $50.68 \mathrm{MHz}$ for ${ }^{15} \mathrm{~N}$ ) in $\mathrm{DMSO}^{-} d_{6}$ ${ }^{1} \mathrm{H}$ and ${ }^{13} \mathrm{C}$ chemical shifts are given on the $\delta$ scale $(\mathrm{ppm})$ and are referenced to internal $\operatorname{TMS}(\delta=0.0) .{ }^{15} \mathrm{~N}$ chemical shifts were referred to external neat $\mathrm{CH}_{3} \mathrm{NO}_{2}$ in a co-axial capillary $(\delta=0.0)$. All 2D experiments (gradient-selected (gs)-COSY, gs-TOCSY, gs-NOESY, gs-HMQC, gs-HMQC-TOCSY,gs-HMQC-RELAY, gs-HMBC) ${ }^{17-19}$ were performed using manufacturer's software (TOPSPIN 3.5). The electrospray mass spectra (ESI-MS) were recorded using an amaZon X ion-trap mass spectrometer (Bruker Daltonics, Bremen, Germany) equipped with an electrospray ion source. All experiments were conducted in both positive and negative polarity mode. Individual samples (with a concentration of $500 \mathrm{ng} \cdot \mathrm{mL}^{-1}$ ) were infused into the ESI source as methanol/water $(1: 1, v / v)$ solutions via a syringe pump with a constant flow rate of $3 \mu \mathrm{L} \cdot \mathrm{min}^{-1}$. The other instrumental conditions were as follows: electrospray voltage of $\pm 4.2 \mathrm{kV}$, capillary exit voltage of $\pm 140 \mathrm{~V}$, drying gas temperature of $220^{\circ} \mathrm{C}$, drying gas flow of $6.0 \mathrm{dm}^{3} \cdot \mathrm{min}^{-1}$, nebulizer pressure of 8.0 psi. Nitrogen was used as the nebulizing and drying gases for all experiments. Tandem mass spectra were collected using collisioninduced dissociation (CID) with $\mathrm{He}$ as the collision gas after isolating of the required ions. Column chromatography was carried out on silica gel (Merck, grade 60, 70-230 mesh) using successive mixtures of chloroform/ethanol (in ratios from 99:1 to 8:2) (S1) or benzene/ethyl acetate (in ratios from 99:1 to 8:2) (S2). Reactions as well as the course of separation and also the purity of all substances were monitored by TLC (elution systems benzene/ethyl acetate (4:1) (S3), chloroform/ethanol (9:1 and 1:1) (S4 and S5), and chloroform/ethyl acetate (7:3) (S6) on Alugram ${ }^{\circledast}$ SIL G/UV 254 foils (Macherey-Nagel). Elemental analyses (C, H, $\mathrm{N})$ were performed with a EA Flash EA 1112 Elemental Analyzer (Thermo Fisher Scientific).

3-Hydroxyethylamino-3-methylqinoline-2,4-dione (2a). Compound was prepared in 45\% yield from 1 a by the procedure described in Ref. ${ }^{10}$. Colorless solid, mp 120-129 ${ }^{\circ} \mathrm{C}$ (benzene); IR: 3312, 3135, 3073, 2981, 2916, 2865, 1711, 1677, 1613, 1485, 1442, 1373, 1346, 1290, 1255, 1195, 1161, 1127, 1072, 962, 887, 793, 760, 695, $665,527,467 \mathrm{~cm}^{-1}$. ESI-MS (pos.) $\mathrm{m} / z(\%): 491.3[2 \cdot \mathrm{M}+\mathrm{Na}]^{+}(42), 257.0[\mathrm{M}+\mathrm{K}]^{+}(78), 235.0[\mathrm{M}+\mathrm{Na}]^{+}(100) . \mathrm{ESI}-$ $\mathrm{MS}$ (neg.) $\mathrm{m} / \mathrm{z}$ (\%): 232.8 [M-H] $]^{-}$(100).For $\mathrm{C}_{12} \mathrm{H}_{14} \mathrm{~N}_{2} \mathrm{O}_{3}$ (234.25) calcd.: C 61.53, H 6.02, N 11.96; found: C 61.50, HG 6.15, N 11.75 .

General procedure for the reaction of compounds $\mathbf{2}$ with isothiocyanic acid. The solution of compound $\mathbf{2}$ ( 1 $\mathrm{mmol}$ ) and potassium thiocyanate $(6 \mathrm{mmol})$ in $\mathrm{AcOH}(3 \mathrm{~mL})$, was stirred for $c .4 \mathrm{~h}$ at $50{ }^{\circ} \mathrm{C}$. The solution was poured onto crushed ice $(60 \mathrm{~mL})$, the resulting precipitate was filtered with suction and crystallized. In the case of oily product, the mixture was extracted with chloroform $(3 \times 20 \mathrm{~mL})$, the collected extracts were evaporated to dryness and chromatographed on silica gel column.

3a-Benzyl-9b-hydroxy-3-(2-hydroxyethyl)-2-thioxo-3,3a,5,9b-tetrahydro-1H-imidazo[4,5-c]quinolin-4(2H)one (3c). Compound was prepared from $2 \mathrm{c}$ in $9 \%$ yield. Colorless solid, mp $236-246^{\circ} \mathrm{C}$ (ethyl acetate). IR: 3199 , 2997, 2931, 1682, 1602, 1495, 1479, 1448, 1404, 1311, 1240, 1191, 1137, 1097, 1069, 1010, 960, 868, 835, $763,749,702,670,627,598,563,546,529 \mathrm{~cm}^{-1}$. ESI-MS (pos.) $\mathrm{m} / z$ (\%): $761.2[2 \cdot \mathrm{M}+\mathrm{Na}]^{+}(23), 408.1[\mathrm{M}+\mathrm{K}]^{+}$ (14), $392.0[\mathrm{M}+\mathrm{Na}]^{+}$(100). ESI-MS (neg.) $\mathrm{m} / \mathrm{z}$ (\%): 737.0 [2.M-H] $^{-}$(25), $403.9[\mathrm{M}+\mathrm{Cl}]^{-}$(18), $367.9[\mathrm{M}-\mathrm{H}]^{-}(100)$. For $\mathrm{C}_{19} \mathrm{H}_{19} \mathrm{~N}_{3} \mathrm{O}_{3} \mathrm{~S}$ (369.44) calcd. C 61.77, $\mathrm{H}$ 5.18, N 11.37, S 8.68; found: C 61.99, H 5.16, N 11.33, S 8.54.

3-(2-Hydroxyethyl)-2-thioxo-2,3-dihydro-1H-imidazo[4,5-c]quinolin-4(5H)-one (4c). Compound was prepared from $2 \mathrm{c}$ in $16 \%$ yield.Yellowish solid, $\mathrm{mp}>330^{\circ} \mathrm{C}$ (methanol). IR: 3418, 3109, 3000, 2891, 2682, 1658, 1613, 
$1571,1521,1502,1464,1440,1422,1373,1346,1287,1259,1196,1151,1051,1035,978,877,854,788,757$, 685, 604, $537 \mathrm{~cm}^{-1}$. ESI-MS (pos.) $\mathrm{m} / z(\%): 545.1[2 \cdot \mathrm{M}+\mathrm{Na}]^{+}$(9), $284.0[\mathrm{M}+\mathrm{Na}]^{+}(100)$. ESI-MS (neg.) $\mathrm{m} / z(\%):$ $542.9[2 \cdot \mathrm{M}-2 \cdot \mathrm{H}+\mathrm{Na}]^{-}$(11), $259.8[\mathrm{M}-\mathrm{H}]^{-}$(100). Calcd. for $\mathrm{C}_{12} \mathrm{H}_{11} \mathrm{~N}_{3} \mathrm{O}_{2} \mathrm{~S}$ (261.30) C 55.16, H 4.24, N 16.08, S 12.27; found: C 55.30, H 4.10, N 15.97, S 12.06 .

2-(2-Hydroxyethyl)-1-methyl-3-thioxo-2,3-dihydroimidazo[1,5-c]quinazolin-5(6H)-one (5a). Compound was prepared from 2 a in $49 \%$ yield. Yellowish solid, $\mathrm{mp} 290-294^{\circ} \mathrm{C}$ (acetic acid). IR: 3389, 3225, 3166, 3101, 3070, 2998, 2938, 1721, 1642, 1612, 1589, 1492, 1453, 1376, 1357, 1303, 1263, 1224, 1159, 1133, 1093, 1053, 917, $863,832,817,760,740,699,666,565,524 \mathrm{~cm}^{-1}$. ESI-MS (pos.) $\mathrm{m} / z$ (\%): $573.1[2 \cdot \mathrm{M}+\mathrm{Na}]^{+}(81), 314.0[\mathrm{M}+\mathrm{K}]^{+}$

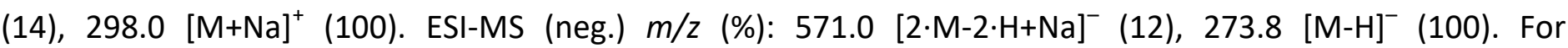
$\mathrm{C}_{13} \mathrm{H}_{13} \mathrm{~N}_{3} \mathrm{O}_{2} \mathrm{~S}$ (275.33) calcd. C 56.71, H 4.76, N 15.26, S 11.65; found: C 56.55, H 4.72, N 14.99, S 11.49.

Table 3. ${ }^{1} \mathrm{H},{ }^{13} \mathrm{C}$ and ${ }^{15} \mathrm{~N}$ NMR data of compounds 7a-d in DMSO- $d_{6}$

\begin{tabular}{|c|c|c|c|c|c|c|c|c|}
\hline & \multicolumn{2}{|c|}{$7 a$} & \multicolumn{2}{|c|}{$7 b$} & \multicolumn{2}{|c|}{$7 c$} & \multicolumn{2}{|c|}{$7 d$} \\
\hline & $\delta_{H}$ & $\delta_{c}$ & $\delta_{H}$ & $\delta_{c}$ & $\delta_{H}$ & $\delta_{c}$ & $\delta_{H}$ & $\delta_{c}$ \\
\hline 1 & 11.28 & $-261.4^{a}$ & 11.39 & $-260.2^{a}$ & 11.37 & & 11.43 & $-261.2^{a}$ \\
\hline 2 & - & 150.4 & - & 150.4 & - & 150.4 & - & 150.9 \\
\hline 3 & - & $-224.7^{a}$ & - & $-222.6^{a}$ & - & - & - & $-223.6^{a}$ \\
\hline 4 & - & 162.2 & - & 162.7 & - & 162.2 & - & 162.3 \\
\hline $4 a$ & - & 115.0 & - & 114.0 & - & 113.9 & - & 114.1 \\
\hline 5 & 7.92 & 127.4 & 7.90 & 127.4 & 7.93 & 127.4 & 7.92 & 127.4 \\
\hline 6 & 7.19 & 122.4 & 7.18 & 122.4 & 7.16 & 122.4 & 7.18 & 122.4 \\
\hline 7 & 7.64 & 134.9 & 7.64 & 134.9 & 7.64 & 134.9 & 7.64 & 134.9 \\
\hline 8 & 7.16 & 114.0 & 7.15 & 115.0 & 7.16 & 115.0 & 7.15 & 115.0 \\
\hline $8 a$ & - & 139.5 & - & 139.6 & - & 139.5 & - & 139.6 \\
\hline $\mathrm{N}(3) \mathrm{CH}_{2}$ & 3.95 & 39.8 & 3.96 & 39.9 & 3.97 & 39.8 & 4.11 & 40.0 \\
\hline $\mathrm{CH}_{2}$ & 3.29 & 36.4 & 3.31 & 36.2 & 3.34 & 36.4 & 3.35 & 37.2 \\
\hline $\mathrm{NH}$ & 7.92 & $267.6^{a, b}$ & 7.86 & $267.6^{a, c}$ & 8.13 & - & 8.56 & $-272.8^{a, d}$ \\
\hline$C=O$ & - & 169.4 & - & 172.3 & - & 170.3 & - & 166.6 \\
\hline $1^{\prime}\left(R^{2}\right)$ & 1.71 & 22.6 & 1.94 & 35.2 & 3.31 & 42.3 & - & 134.9 \\
\hline $2^{\prime}\left(R^{2}\right)$ & - & - & 1.37 & 27.3 & - & 136.3 & 7.71 & 127.2 \\
\hline $3^{\prime}\left(R^{2}\right)$ & - & - & 1.16 & 21.8 & 7.16 & 128.1 & 7.42 & 128.2 \\
\hline $4^{\prime}\left(R^{2}\right)$ & - & - & 0.79 & 13.8 & 7.23 & 129.1 & 7.48 & 131.0 \\
\hline $5^{\prime}\left(R^{2}\right)$ & - & - & - & - & 7.16 & 126.2 & - & - \\
\hline
\end{tabular}

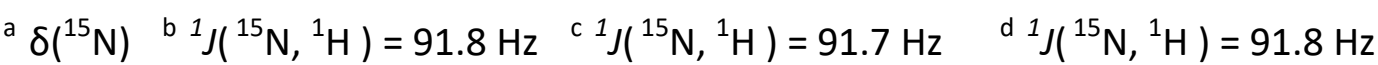

1-Butyl-2-(2-hydroxyethyl)-3-thioxo-2,3-dihydroimidazo[1,5-c]quinazolin-5(6H)-one (5b). Compound was prepared from $\mathbf{2 b}$ in $42 \%$ yield. Yellowish solid, $\mathrm{mp} 241-245{ }^{\circ} \mathrm{C}$ (ethyl acetate); IR: 3371, 3143, 3099, 3062, 2993, 2932, 2887, 1726, 1633, 1615, 1592, 1494, 1461, 1431, 13988, 1289, 1279, 1239, 1204, 1155, 1135, 1092, 1063, 1017, 964, 861, 824, 791, 752, 741, 663, 574, $564 \mathrm{~cm}^{-1}$. ESI-MS (pos.) m/z (\%): $657.2[2 \cdot \mathrm{M}+\mathrm{Na}]^{+}$ $(37), 495.6[3 \cdot \mathrm{M}+\mathrm{H}+\mathrm{K}]^{+}(16), 356.0[\mathrm{M}+\mathrm{K}]^{+}(12), 340.0[\mathrm{M}+\mathrm{Na}]^{+}(100), 318.1[\mathrm{M}+\mathrm{H}]^{+}(6) . \mathrm{ESI}-\mathrm{MS}$ (neg.) $\mathrm{m} / z(\%)$ : $655.1[2 \cdot \mathrm{M}-2 \cdot \mathrm{H}+\mathrm{Na}]^{-}$(6), $315.9[\mathrm{M}-\mathrm{H}]^{-}$(100). For $\mathrm{C}_{16} \mathrm{H}_{19} \mathrm{~N}_{3} \mathrm{O}_{2} \mathrm{~S}$ (317.41) calcd. C 60.54, H 6.03, N 13.24, S 10.10; found: C 60.46, H 6.02, N 13.25, S 10.22. 
2-(2-Hydroxyethyl)-1-phenyl-3-thioxo-2,3-dihydroimidazo[1,5-c]quinazolin-5(6H)-one (5d). Compound was prepared from $\mathbf{2 d}$ in $26 \%$ yield besides $6 \mathrm{~d}$. Yellowish solid, $\mathrm{mp} 248-252^{\circ} \mathrm{C}$ (ethyl acetate). IR: $3378,3254,3206$, 2994, 2930, 2876, 1732, 1645, 1616, 1591, 1485, 1443, 1380, 1325, 1296, 1262, 1232, 1186, 1125, 1080, 1047, 1022, 928, 846, 789, 745, 709, 669, 585, $506 \mathrm{~cm}^{-1}$. ESI-MS (pos.) $\mathrm{m} / \mathrm{z}$ (\%): $697.1[2 \cdot \mathrm{M}+\mathrm{Na}]^{+}(22), 525.6$ $\left.[3 \cdot \mathrm{M}+\mathrm{H}+\mathrm{K}]^{+}(9), 376.0{ }^{\mathrm{M}+\mathrm{K}}\right]^{+}(12), 360.0$ [M+Na] $^{+}(100)$. ESI-MS (neg.) $\mathrm{m} / \mathrm{z}(\%): 695.0[2 \cdot \mathrm{M}-2 \cdot \mathrm{H}+\mathrm{Na}]^{-}(7), 335.9$ $[\mathrm{M}-\mathrm{H}]^{-}$(100). For $\mathrm{C}_{18} \mathrm{H}_{15} \mathrm{~N}_{3} \mathrm{O}_{2} \mathrm{~S}$ (337.40) calcd. C 64.08, H 4.48, N 12.45, S 9.50; found: C 64.25, H 4.39, N 12.26, S 9.39 .

3-(2-hydroxyethyl)-3a-phenyl-2-thioxo-3,3a-dihydro-2H-imidazo[4,5-c]quinolin-4(5H)-one (6d). Compound was prepared from $\mathbf{2 d}$ in $8 \%$ yield besides $\mathbf{5 d}$. Colorless solid, mp $232-236^{\circ} \mathrm{C}$ (ethyl acetate); IR: 3426,3204 , 2993, 2930, 1731, 1680, 1599, 1485, 1445, 1401, 1366, 1246, 1196, 1157, 1140, 1079, 1037, 1005, 977, 949, 936, 901, 788, 760, 745, 698, 652, 588, 558, $518 \mathrm{~cm}^{-1}$. ESI-MS (pos.) $\mathrm{m} / z$ (\%): $697.2[2 \cdot \mathrm{M}+\mathrm{Na}]^{+}(8), 525.6$ $[3 \cdot \mathrm{M}+\mathrm{H}+\mathrm{K}]^{+}(9), 376.0[\mathrm{M}+\mathrm{K}]^{+}(13), 360.0[\mathrm{M}+\mathrm{Na}]^{+}(100), 338.1[\mathrm{M}+\mathrm{H}]^{+}$(13). ESI-MS (neg.) $\mathrm{m} / \mathrm{z}(\%): 695.0$ [2.M$2 \cdot \mathrm{H}+\mathrm{Na}]^{-}$(6), 335.9 [M-H] $]^{-}$(100). For $\mathrm{C}_{18} \mathrm{H}_{15} \mathrm{~N}_{3} \mathrm{O}_{2} \mathrm{~S}$ (337.40) calcd.: C 64.08, H 4.48, N 12.45, S 9.50; found: C $64.01, \mathrm{H} 4.42$, N 12.20, S 9.45 .

General procedure for the reaction of chloroderivatives 1a-d with ethylene diamine. To a solution of compound 1 (20 mmol) in DMF $(125 \mathrm{~mL})$ was added potassium carbonate $(45 \mathrm{mmol})$ and ethylene diamine $(1.6 \mathrm{~mL}, 2.4 \mathrm{mmol})$. The mixture was stirred at $\mathrm{rt}$ for $4 \mathrm{~h}$, then poured onto crushed ice $(1000 \mathrm{~mL}) \mathrm{and}$ extracted with chloroform $(10 \times 40 \mathrm{~mL}$ ). Collected extracts were evaporated to dryness and crystallized from appropriate solvent or chromatographed on silica gel column.

$\mathbf{N}$-(2-(2,4-Dioxo-1,2-dihydroquinazolin-3(4H)-yl)ethyl)acetamide (7a). Compound was prepared from 1a in $52 \%$ yield. Colorless solid, $\mathrm{mp}$ 271-281 ${ }^{\circ} \mathrm{C}$ (ethanol). IR: 3293, 3090, 2999, 2936, 1712, 1655, 1603, 1567, 1491, $1454,1409,1380,1341,1283,1266,1234,1177,1163,1108,1047,1026,927,819,765,694,682,666,634$, 599, 539, $466 \mathrm{~cm}^{-1}$. ESI-MS (pos.) $\mathrm{m} / \mathrm{z}(\%): 517.2[2 \cdot \mathrm{M}+\mathrm{Na}]^{+}(71), 286.0[\mathrm{M}+\mathrm{K}]^{+}(11), 270.0[\mathrm{M}+\mathrm{Na}]^{+}(100) . \mathrm{ESI}-$

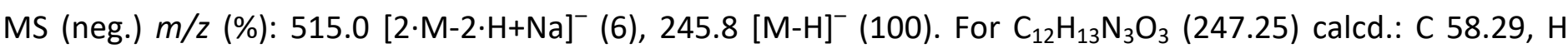
5.30, N 16.99; found: C 58.26, H 5.41, N 16.91 .

$\mathbf{N}$-(2-(2,4-Dioxo-1,2-dihydroquinazolin-3(4H)-yl)ethyl)pentanamide (7b). Compound was prepared from $\mathbf{1 b}$ in $36 \%$ yield. Colorless solid, $\mathrm{mp} 208-210^{\circ} \mathrm{C}$ (benzene). IR :3309, 3198, 3074, 2936, 1715, 1671, 1548, 1518, 1493, $1455,1432,1407,1374,1653,1330,1278,1231,1172,1123,1052,1028,940,868,824,756,694,680,597$, 560, $528 \mathrm{~cm}^{-1}$. ESI-MS (pos.) $\mathrm{m} / z(\%): 601.3[2 \cdot \mathrm{M}+\mathrm{Na}]^{+}(35), 328.1[\mathrm{M}+\mathrm{K}]^{+}(13), 312.1[\mathrm{M}+\mathrm{Na}]^{+}(100), 309.1$ $[2 \cdot \mathrm{M}+\mathrm{H}+\mathrm{K}]^{+}(12), 290.1[\mathrm{M}+\mathrm{H}]^{+}$(5). ESI-MS (neg.) $\mathrm{m} / \mathrm{z}$ (\%): $599.1[2 \cdot \mathrm{M}-2 \cdot \mathrm{H}+\mathrm{Na}]^{-}$(6), $287.9[\mathrm{M}-\mathrm{H}]^{-}(100)$. For $\mathrm{C}_{15} \mathrm{H}_{19} \mathrm{~N}_{3} \mathrm{O}_{3}$ (289.33) calcd: C 62.27, H 6.62, N 14.52; found: C 62.00, H 6.60, N 14.38.

$\mathbf{N}$-(2-(2,4-Dioxo-1,2-dihydroquinazolin-3(4H)-yl)ethyl)-2-phenylacetamide (7c). Compound was prepared from $1 \mathrm{c}$ in $32 \%$ yield. Colorless solid, $\mathrm{mp} 255-258{ }^{\circ} \mathrm{C}$ (ethanol).IR: 3307, 3196, 3065, 2909, 1723, 1657, 1608, $1546,1494,1453,1412,1382,1339,1290,1266,1247,1201,1176,1160,1073,1036,1025,952,834,790$, $758,732,703,692,665,561,533,523,464 \mathrm{~cm}^{-1}$ ESI-MS (pos.) $\mathrm{m} / z$ (\%): $669.2[2 \cdot \mathrm{M}+\mathrm{Na}]^{+}(19), 362.0[\mathrm{M}+\mathrm{K}]^{+}$

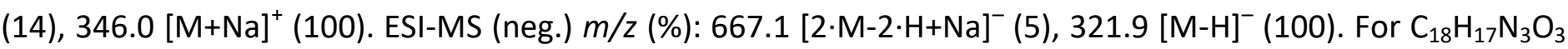
(323.35) calcd. C 66.36, H 5.30, N 13.00; found: C 66.22, H 5.36, N 12.92.

$\mathbf{N - ( 2 - ( 2 , 4 - d i o x o - 1 , 2 - d i h y d r o q u i n a z o l i n - 3 ( 4 H ) - y l ) e t h y l ) b e n z a m i d e ~ ( 7 d ) . ~ C o m p o u n d ~ w a s ~ p r e p a r e d ~ f r o m ~ 1 d ~ i n ~}$ $7 \%$ yield. Colorless solid, $\mathrm{mp} 249-253^{\circ} \mathrm{C}$ (ethyl acetate). IR: 3324, 3064, 3006, 2940, 1721, 1657, 1604, 1579, $1546,1492,1453,1411,1382,1339,1289,1267,1232,1175,1153,1060,1019,938,852,801,764,733,693$, $682,666,560,532 \mathrm{~cm}^{-1}$. ESI-MS (pos.) $\mathrm{m} / \mathrm{z}(\%): 641.1$ [2.M+Na] $^{+}(35), 348.0[\mathrm{M}+\mathrm{K}]^{+}(14), 322.0[\mathrm{M}+\mathrm{Na}]^{+}(100)$. ESI-MS (neg.) $\mathrm{m} / z$ (\%): 307.9 [M-H] $]^{-}$(100). For $\mathrm{C}_{17} \mathrm{H}_{15} \mathrm{~N}_{3} \mathrm{O}_{3}$ (309.32) calcd. C 66.01; H, 4.89; N, 13.58; found: C 65.96, H 4.90, N 13.43. 
10b-Benzoyl-1,2,3,10b-tetrahydroimidazo[1,2-c]quinazolin-5(6H)-one (8d). Compound was prepared from 1d in $25 \%$ yield besides $7 d$. Colorless solid, $\mathrm{mp} 217-226^{\circ} \mathrm{C}$ (ethyl acetate); IR: 3277, 3196, 3124, 3061, 2982, 2891, $1678,1597,1508,1486,1478,1467,1447,1331,1309,1264,1228,1173,1159,1131,1118,1082,940,908$, $879,866,833,792,757,707,691,663,639 \mathrm{~cm}^{-1}$. ESI-MS (pos.) $\mathrm{m} / z(\%): 609.1[2 \cdot \mathrm{M}+\mathrm{Na}]^{+}(27), 587.1[2 \cdot \mathrm{M}+\mathrm{H}]^{+}$ $(16), 459.6[3 \cdot \mathrm{M}+\mathrm{H}+\mathrm{K}]^{+}(10), 332.0[\mathrm{M}+\mathrm{K}]^{+}(8), 316.0[\mathrm{M}+\mathrm{Na}]^{+}(53), 294.0[\mathrm{M}+\mathrm{H}]^{+}(100) . \mathrm{ESI}-\mathrm{MS}$ (neg.) $\mathrm{m} / z(\%):$ 291.9 [M-H] $]^{-}$(100). For $\mathrm{C}_{17} \mathrm{H}_{15} \mathrm{~N}_{3} \mathrm{O}_{2}$ (293.32) calcd.: C 69.61, H 5.15, N 14.33; found: C 69.62, H 5.25, N 14.27.

\section{Reduction of compound $8 \mathrm{~d}$.}

10b-(Hydroxy(phenyl)methyl)-1,2,3,10b-tetrahydroimidazo[1,2-c]quinazolin-5(6H)-one (9d). To the solution of compound $8 \mathbf{d}(0.2051 \mathrm{~g}, 0.7 \mathrm{mmol})$ in methanol $(10 \mathrm{~mL}), \mathrm{NaBH}_{4}(0.029 \mathrm{~g}, 0.78 \mathrm{mmol})$ was added during 5 min. After $1 \mathrm{~h}$ at $\mathrm{rt}$, the solution was poured onto crushed-ice. Hydrochloric acid $(0.5 \mathrm{~mL})$ and, after $10 \mathrm{~min}$, the solution of sodium bicarbonate $(6 \%, 12 \mathrm{~mL})$ were added. The mixture was extracted $(8 \times 25 \mathrm{~mL})$ of chloroform. Collected extracts were dried with sodium sulfate, evaporated to dryness and crystallized from

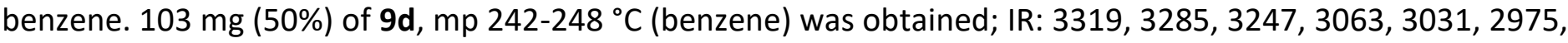
$2892,1666,1614,1598,1510,1487,1448,1337,1308,1259,1200,1172,1156,1135,1115,1096,1058,1039$, 1025, 988, 959, 892, 869, 823, 787, 755, 709, 680, 610, 586, $535 \mathrm{~cm}^{-1}$. ESI-MS (pos.) m/z (\%): 318.1 [M+Na] (28), $296.1[\mathrm{M}+\mathrm{H}]^{+}$(100). For $\mathrm{C}_{17} \mathrm{H}_{17} \mathrm{~N}_{3} \mathrm{O}_{2}$ (295.34) calcd.: C 69.14, H 5.80, N 14.23; found: C 69.24, H 6.05, N 14.13.

\section{Acknowledgements}

A.K. and M.R. grateful for the financial support from the internal grant of TBU in Zlin No. IGA/FT/2019/010, funded from the resources of specific university research. The authors would also like to thank Mrs. $H$. Geržová (Faculty of Technology, Tomas Bata University in Zlín) for her technical assistance.

Conflict of interest: The authors have declared that they have no conflict of interest.

Author contribution statement: $A$. K. designed the studies and wrote the manuscript. A. L. and M. R. conducted the experiments.

\section{References}

1. Kafka, S.; Klásek, A.; Polis J.; Košmrlj, J. Heterocycles 2002, 57, 1659.

https://doi.org/10.3987/COM-02-9522

2. Podesva, C.; Vagi, K.; Solomon, C. Can. J. Chem. 1968, 46, 2263.

3. Stadlbauer, W.; Lutschounig, H. K.; Schinder, G.; Witoszynskij, T.; Kappe, T. J. Heterocyclic Chem. 1992, 29, 1535.

4. Shin, S.; Kang, H.; Choi, L.; Jung, K. Neuroscience 2013, 232, 1.

https://doi.org/10.1016/i.neuroscience.2012.12.008

5. Cifuentes-Pagano, E. M. Meijles, D. N.; Pagano, P. J. Curr. Pharm. Design, 2015, 21, 6023. https://doi.org/10.2174/1381612821666151029112013

6. Mittal, R.; Debs, L. H. ; Nguyen, D.; Patel, A. P.; Grati, M.; Mittal, J.; Yan, D.; Eshraghi, A. A.; Liu, X. Z. J. Cellular Physiol, 2017, 232, 2710.

https://doi.org/10.1002/icp.25695

7. Klásek, A..; Kořistek, K.; Lyčka, A.; Holčapek, M. Tetrahedron 2003, 59, 1283. 
8. Klásek, A..; Kořistek, K.; Lyčka, A.; Holčapek, M. Tetrahedron 2003, 59, 5279.

9. Mrkvička, V.; Rudolf, O.; Lyčka, A.; Klásek, A. Tetrahedron 2011, 67, 2407. https://doi.org/10.1016/i.tet.2011.02.002

10. Klásek, A.; Křemen, F.; Křemenová, H.; Lyčka, A.; Rouchal, M. Tetrahedron 2017, 73, 1583. https://doi.org/10.1016/j.tet.2017.02.007

11. Rudolf, O.; Mrkvička, V.; Lyčka, A.; Rouchal, A.;Klásek, A. Helv. Chim. Acta 2012, 95, 1352." https://doi.org/10.1002/hlca.201200049

12. Herndon, J. L.; Ismaiel, A.; Igher, S. P.; Teitler, I.; Richard, A.; Glennon, X. J. Med. Chem. 1992, 35, 4903. https://doi.org/10.1021/jm00104a017

13. Borgions, F.; Ghyssels, D.; Van Aerschot; A., Rozenski; J., Herdewijn, P. Helv. Chim. Acta 2006, 89, 1194. https://doi.org/10.1002/hlca.200690118

14. Theodorou, V.; Paraskevopoulos, G.; Skobridis, K. Arkivoc 2015, (vii), 101.

15. Vaughn, H. L.; Robbins, M. D. J. Org. Chem. 1975, 40, 1188. https://doi.org/10.1021/jo00896a050

16. Szyszkovwska, A.; Klasek, A.; Pawledzio, S.; Trzybinski, D.; Wozniak, K.; Zarzyka, I. J. Mol. Struct.2018, 1153, 230.

https://doi.org/10.1016/i.molstruc.2017.10.014

17. Berger, S.; Braun S. 200 and More Basic NMR Experiments: A Practical Course, 3rd Ed. Weinheim: WileyVCH; 2004.

18. Claridge, T. D. W. High-Resolution NMR Techniques in Organic Chemistry, 3rd Edition. Elsevier, 2016. https://doi.org/10.1016/B978-0-08-099986-9.00002-6

19. Marek, R.; Lyčka, A.; Kolehmainen, E.; Sieveren, E.; Toušek, J. Cur. Org. Chem. 2007, 11, 1154. https://doi.org/10.2174/138527207781662519 